\title{
Deep Learning Approaches for Tomato Plant Disease Detection
}

\author{
Md Abdur Rahman \\ Associate Professor of Computer Science, Department of Mathematics, \\ Jahangirnagar University, Savar, Dhaka, Bangladesh \\ marahmanju@juniv.edu
}

\begin{abstract}
Agriculture is the mainstream to keep pace in the Bangladeshi economy. Plant disease became a threat to food security as it is a very important factor to deteriorate the quality and quantity of harvest. Therefore, it is important to detect the plant diseases early which results in interrupting from falling the massive destruction of harvest. But, an erroneous diagnosis of the disease results in the inappropriate use of pesticides. To enhance the production quality and quantity, a deep learning-based approach is proposed to detect the tomato leaf diseases, and then classify the types of the disease using an image dataset. This proposed approach trained two model architectures: inception V3 and Convolutional Neural Network (CNN). Inception V3 performs well and reaches a success rate of $96.11 \%$ to identify whether the specific plant leaf is infected or healthy. The success rate is significant and makes this approach a very useful way or early forewarning tool, and this approach might be an essential system to operate real agriculture fields. As the detection accuracy is recorded as $94.72 \%$ for CNN. We confirm that it achieves the experimental results with $94.72 \%$ and $96.11 \%$ for the detection and classification of infected leaves from the dataset for CNN and Inception $\mathrm{V} 3$ respectively.
\end{abstract}

Keywords: Deep learning, Convolutional Neural Network (CNN), Inception model, Transfer learning, PoolinglLayer, Softmax layer

\section{Introduction}

The efficient research to plant disease detection and classification is closely connected to sustainable agriculture as climate change is responsible for the development of pathogen with altering phases and degrees [1]; host resistance is modified with changing the climate. As a result, it leads to physiological changes for the interactions of the pathogen [2][3]. However, the situation is becoming more vulnerable as it is supposed to transfer diseases globally than before. These new diseases are observed in new locations in which these are completely unknown and unidentified, and no expertise can be able to identify them to take the measures [4][5][6]. Many researchers used deep learning approaches for solving complex tasks in different research areas in biomedicine, bioinformatics, biology, and robotics [7][8][9][10]. One framework named Caffe was used to develop a CNN for several class classifications [11].

Article history:

Received (July 4, 2020), Review Result (August 7, 2020), Accepted (September 8, 2020)

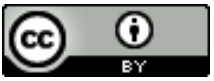

C 2020 Md Abdur Rahman. Published by Global Vision Press

This is an open access article distributed under the terms of the Creative Commons Attribution License (CC BY4.0), which permits unrestricted use, distribution, and reproduction in any medium, provided the original author and source are credited. 
A CNN with ReLu activation is proposed for binary classification along with a normalization layer regarding local contrast [12].

Also, GoogleNet and AlexNet are efficient to train and fine-tune for the classification of infected locations and symptoms of plant leaves [13]. Another work proposed three stage training using CNN [14]. During the first to three stages, it realizes with lesions presence, to generate heat map to detect infection, and ultimately, features map are created. The saliency map method of Brahimi et al [15] to localize infected regions is supposed to improve accuracy for classification. Wang et al [16] studied the effect of the depth of neural network on the accuracy for classification. The biological name of tomatoes is Solanum Lycopersicum and this vegetable grows on drained soil [17]. Around $90 \%$ of farmers choose their field to grow it.

The literature survey reveals the necessity to detect and classify plant disease in the field of agriculture, and plant pathology. Agarwal et al. proposed a deep learning model to detect tomato leaf diseases whose average accuracy is $91.2 \%$ [18]. This paper proposes the inception V3 model with $96.1 \%$ accuracy which is higher than the previous work. In this paper, Section 2 describes the different types of diseases for tomato leaves, Section 3 gives the materials and methods, Section 4 presents the results and discussion, and final Section 4 is used to conclude the paper summary as the conclusion.

\section{Tomatoes leaves diseases}

There are different types of diseases for tomato leaves. Two among them are discussed in the following:

\subsection{Early blight}

The fungi Alternariatomatophila and A. solani cause early blight disease in the tomato plant. There are small, and brown lesions on the older foliage on the plants. Spots are enlarged and became concentric rings.

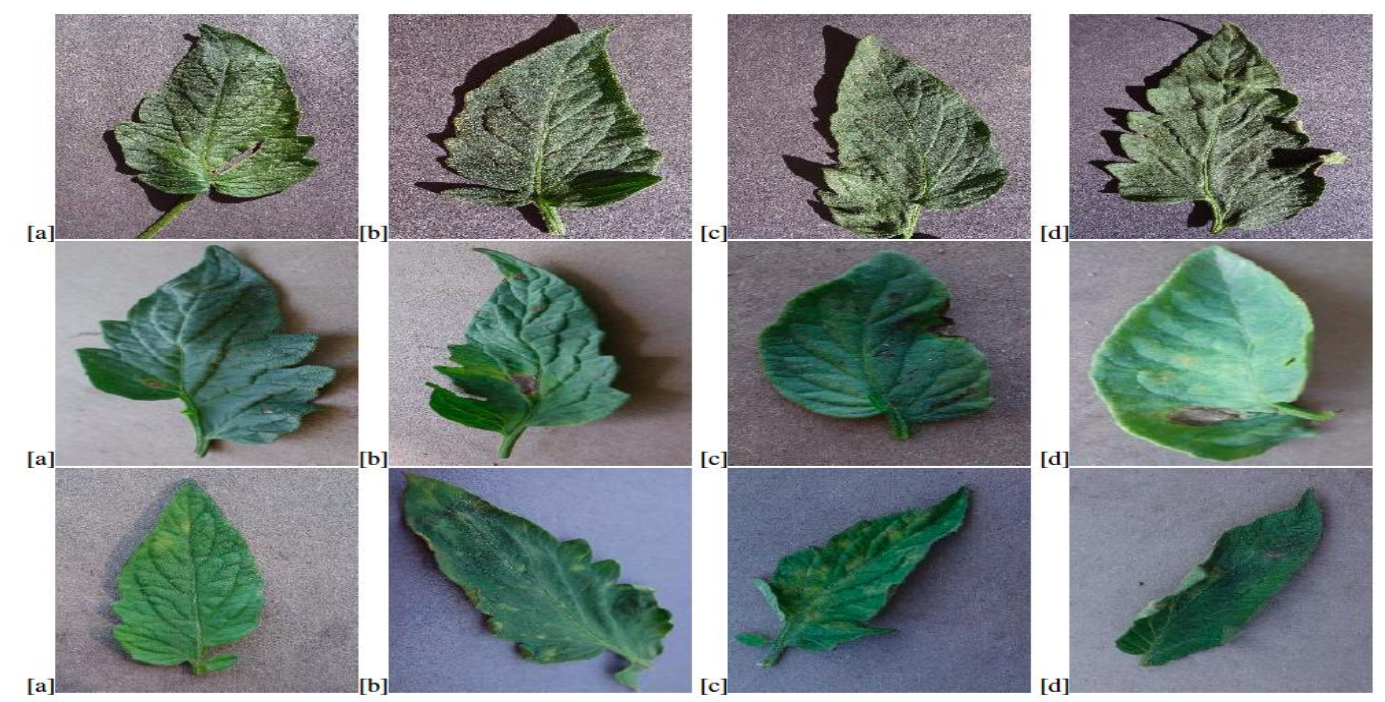

Figure 1. Images confusion shown in healthy (a-d), early blight (e-h), and leaf mold (i-l) of tomato plants from the PlantVillage dataset 
The surrounding of the spots may be becoming yellow. Most foliage may be killed if there are high temperature and humidity at that time. The lesion is observed on the stems. Infected fruits do not last more. [Figure 1] shows the different types of images from the dataset early blight, healthy, late blight, and leaf mold.

\subsection{Leaf mold}

The fungus Passalora fulva causes the disease leaf mold which is observed on older leaves close to the soil. This happens for poor air movement and high humidity. The symptoms are pale green or yellowish spots initially on the top side of the leaf surface, which is enlarging and turning a distinctive yellow.

\section{Materials and methods}

The procedures for developing the model for disease recognition of tomatoes plant are described in the following.

\subsection{Convolutional Neural Network (CNN)}

CNN model has multi-layer along with a supervised learning architecture. The main parts are the feature extractor and classifier. The roles of the feature extractor are retrieving distinguishing features from the raw images. These happen by two operations: convolutional filtering and downsampling. The two vital properties of convolutional filtering are the local receptive field and shared weights. Convolutional filtering works as a local feature extractor which makes the relationships between pixels of training images. As a result, multi-filters extract the most effective and high level features for enhancing the ability of the CNN model for recognition. Moreover, it is important to reduce the number of trainable parameters as well as to improve the efficiency of training which happens by weight sharing and downsampling. The back-propagation algorithm is used to train the classifier in which it learns the weights by the feature extractor.

\subsection{Inception model}

Inception $\mathrm{v} 3$ is becoming popular and accepted widely for image processing as it is a very effective image recognition model. It is made up of max pooling, average pooling, convolutions, dropouts, concats, and fully connected layers. Also, Softmax is used to compute the loss. Machine learning researchers need to spend huge time to construct a neural network for training the model. Sometimes, it may require days or weeks for training the model. This might run a learning process using highly cost hardware, for example, GPUs and parallelized systems. In another way, it is possible to run the same model within seconds if a preconstructed network structure and pretrained weights are used. In this way, learning outputs are transferred among different parties. Furthermore, you don't need to have a large scale training dataset. After transferring learning outcomes, there is no requirement for a large scale training dataset.

\subsection{Dataset}

The final dataset has been made by the images for tomato plant leaves are collected from the PlantVillage dataset as well as the fields of Bangladesh. It contains 1800 image patches: 1300 are infected and 500 are healthy leaves. [Figure 1] shows the sample images of leaves 
for early blight, leaf mold, and healthy leaves. These images are different regard to scale, illumination, rotation and partial viewpoint changes. Sometimes the background, texture, water body, and soil make it difficult to recognize correctly.

Deep learning does not be able to recognize shape and location. The factors above mentioned are responsible for significant challenges for tomato early blight disease recognition. There are no constraints for tomato plant images and their pixels. The patches of $128 \times 128$ pixels in size are chosen to extract from original larger images of the dataset. Also, a moving window of a stride of 96 pixels is used in this approach. Finally, the experts identified the patches which contain lesions to use it as positive samples, and patches that do not contain any lesions are used as negative samples.

\subsection{Proposed framework}

$\mathrm{CNN}$ is made up of multi-layers along with max pooling and dropout and it is a supervised learning architecture. For larger datasets, architectures must be deeper to get better results. We have used the dropout layer to prevent over-fitting. In the proposed approach, we have used two InceptionV3 and $\mathrm{CNN}$ with fine tuning the parameters so that the best performance is achieved. [Figure 2] shows the architecture of the Inception v3 model which is used to classify the three classes. That means that it is developed to identify whether it is infected or healthy. Before training the models, it is required to scale images because the inception model handles pixels between 0 and 128. If there are transfer values already calculated, and then it just is loaded. If it is not calculated and saved to a cache file. The transfer values for the image are calculated using the inception model. The transfer values of healthy leaves are saved in the cache file.

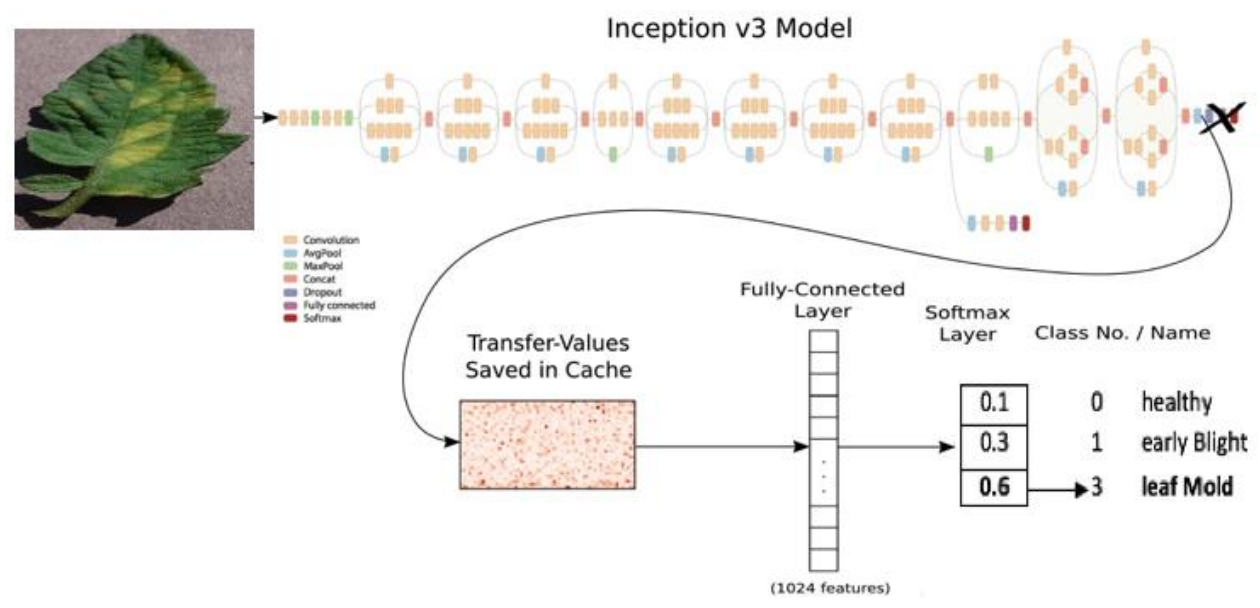

Figure. 2. Architecture of Inception v3 used by the proposed model

\section{Results and discussion}

We have chosen the PlantVillage dataset which is processed and augmented before training the model. Usually, convolutional networks are capable of learning features while training larger datasets, and the output is not satisfactory with the only original images. Therefore, fine-tuning the parameters helps to achieve expected accuracy from the network.

The file path of images has been set up in google drive for the training and testing set to calculate the transfer values and save them into the cache. If there are transfer values already 
calculated, and then it just is loaded. If it is not calculated and saved to a cache file. The transfer values for the image are calculated using the inception model. The transfer values of healthy and infected leaves are saved in the cache file.

On Google collab environment, Python library, Keras is used to implement this proposed model. 78 seconds and 53 seconds for each epoch are required for CNN, and Inception V3 models respectively. After ending each epoch, a slight break happens to compute its loss and accuracy for the deep learning model in terms of testing sample images for validation.

The hidden layer is used to make feature maps in which each neuron of a feature map considers at different locations of the input image. Ultimately, this feature map is generated because of convolution through the image with filters. Each layer has different features stated in a different block. For the feature map, visualization is created for the activation. Activation of the first convolutional layer and the second convolutional layer of the deep network are shown in [Figure 3].
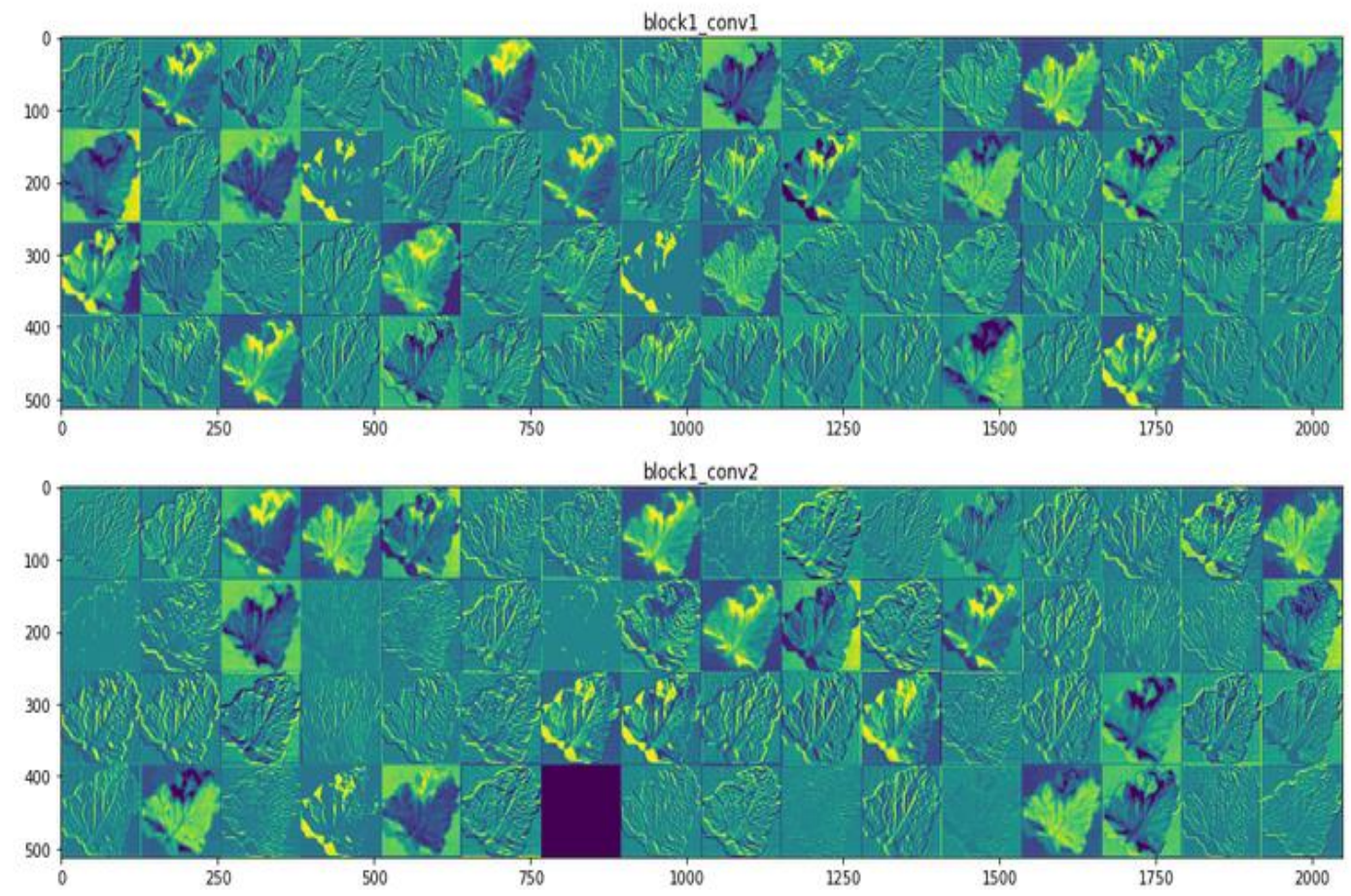

Figure 3. Activation of first convolutional layer and the second convolutional layer of deep network

The green line shows the training accuracy of the network on the training set through iterations in [Figure 4(a)]. On the other hand, the validation accuracy of the network on the validation set expresses the red line. The green line denotes the training loss and the red line expresses validation loss in [Figure 4(b)]. After the 5th epochs training, it achieved about $91 \%$ accuracy with reduced loss. The best performance is recorded in the 22nd epochs which are 96.1\%. This accuracy is far better than the results of Agarwal et al., 2020 which is $91.20 \%$. Even the accuracy of CNN (94.72\%) is larger than Agarwal et al., 2020 presented in [Table 1].

Table 1. Input data

\begin{tabular}{|c|c|c|c|c|c|c|c|}
\hline Model & $\begin{array}{c}\text { Test } \\
\text { Accuracy }\end{array}$ & MSE & MAE & Epochs & $\begin{array}{c}\text { Time } \\
\text { (s/epoch) }\end{array}$ & $\begin{array}{c}\text { Trainable } \\
\text { params }\end{array}$ & $\begin{array}{c}\text { Non- } \\
\text { trainable } \\
\text { params }\end{array}$ \\
\hline
\end{tabular}




\begin{tabular}{|c|c|c|c|c|c|c|c|}
\hline $\begin{array}{c}\text { Agarwal et } \\
\text { al., 2020 }\end{array}$ & 0.912 & -- & -- & -- & -- & 208,802 & 0 \\
\hline CNN & 0.9472 & 0.0470 & 0.0592 & 25 & 78 & $13,391,939$ & 2,880 \\
\hline InceptionV3 & 0.9611 & 0.0202 & 0.0450 & 25 & 53 & $1,313,283$ & $21,802,784$ \\
\hline
\end{tabular}

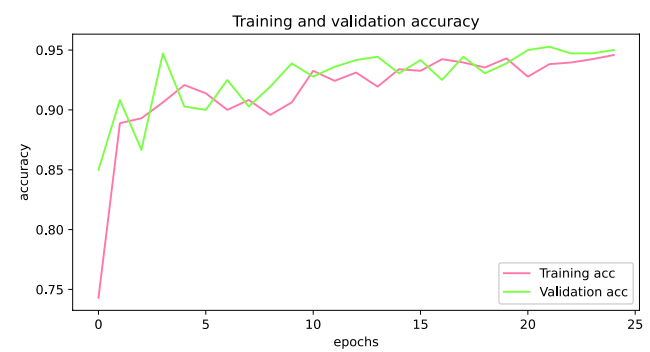

(a)

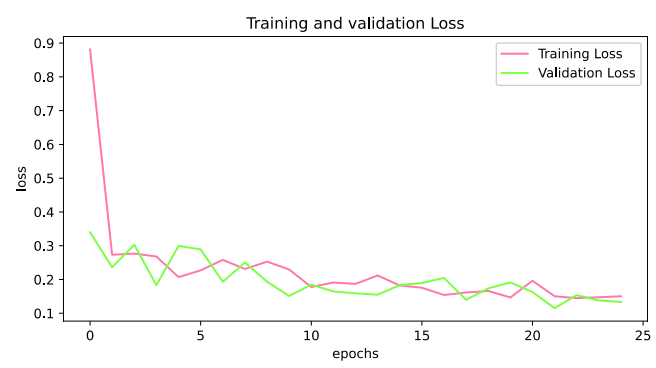

(b)

Figure 4. Plots created by InceptionV3 for (a) the training and validation accuracy, and (b) the training and validation loss

We also focus to find out the reasons for misclassification. That is why misclassification samples are analyzed to identify distinguished things. We observed that blur, water droplets and small or incomplete lesions make the distinguished mistakes. Also, shadow, light spot, water droplets and complex background are responsible for misclassification. However, preprocessing and augmentation before training demonstrate that this proposed model can correctly and effectively recognize almost all of the tomato blast lesions. The results may have slight variation because of the diverse random initialization of the model.

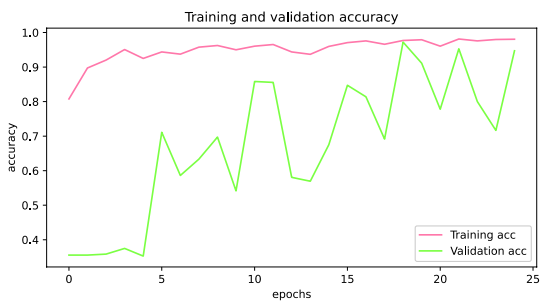

(a)

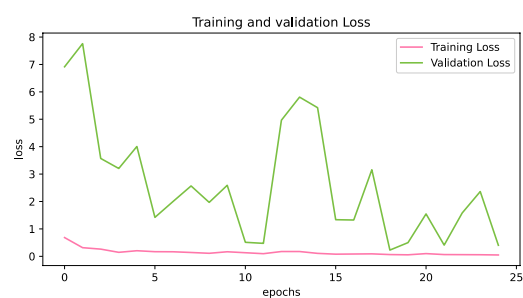

(b)

Figure 5. Plots created by CNN for (a) the training and validation accuracy, and (b) the training and validation loss 


\section{Conclusion}

In this paper, we have studied several deep learning architectures, and two approaches of deep learning with fine-tuning parameters were explored to automatically detect and classify tomato plant diseases. Inception V3 performs well and reaches a success rate of $96.11 \%$ to identify whether the specific plant leaf is infected or healthy. However, the detection and classification accuracy is $94.72 \%$ for $\mathrm{CNN}$ which is lower than the previous model. The success rate of Inception V3 and CNN is significant as it is far better than another work of Agarwal et al., 2020. This result confirms this approach robust and very useful for early forewarning tools, and this approach could be an essential system to operate real agriculture fields. In the future, we will work to identify and classify more diseases for different plants in the same application.

\section{Acknowledgments}

I would like to thank University Grants Commission, Bangladesh as well as Jahangirnagar University, Bangladesh to support this research project with their generous funding and logistic support to complete it successfully.

\section{References}

[1] K. A. Garrett, S. P. Dendy, E. E. Frank, M. N. Rouse, and S. E. Travers, "Climate change effects on plant disease: genomes to ecosystems," Annual Review of Phytopathology, vol.44, pp.489-509, (2006) DOI: 10.1146/annurev.phyto.44.070505.143420

[2] S.M. Coakley, H. Scherm, and S. Chakraborty, "Climate change and plant disease management," Annual Review of Phytopathology, vol.37, no.1, pp.399-426, (1999) DOI: 10.1146/annurev.phyto.37.1.399

[3] S. Chakraborty, A. V. Tiedemann, and P. S. Teng, "Climate change: potential impact on plant diseases," Environmental Pollution, vol.108, no.3, pp.317-326, 2000. DOI: 10.1016/S0269-7491(99)00210-9

[4] A. J. Tatem, D. J. Rogers, and S. I. Hay, "Global transport networks and infectious disease spread," Advances in Parasitology, vol.62, pp.293-343, (2006) DOI: 10.1016/S0065-308X(05)62009-X

[5] J. R. Rohr, T. R. Raffel, J.M. Romansic, H. McCallum, and P. J. Hudson, "Evaluating the links between climate, disease spread, and amphibian declines," Proceedings of the National Academy of Sciences of the United States of America, vol.105, no.45, pp.17436-17441, (2008) https://www.pnas.org/content/105/45/ 17436

[6] T. Van der Zwet, "Present worldwide distribution of fire blight," in Proceedings of the 9th International Workshop on Fire Blight, vol.590, Napier, New Zealand, October (2001) DOI: 10.17660/ActaHortic.2 002.590 .1

[7] I. Lenz, H. Lee, and A. Saxena, "Deep learning for detecting robotic grasps," The International Journal of Robotics Research, vol.34, no.4-5, pp.705-724, (2015) DOI: 10.1177/0278364914549607

[8] B. Alipanahi, A. Delong, M. T. Weirauch, and B. J. Frey, "Predicting the sequence specificities of DNA- and RNA-binding proteins by deep learning," Nature Biotechnology, vol.33, no.8, pp.831-838, (2015) DOI: 10.1504/IJDMB.2017.084265

[9] L. Zhang, G.-S. Xia, T.Wu, L. Lin, and X. C. Tai, "Deep learning for remote sensing image understanding," Journal of Sensors, vol.2016, Article ID 7954154, pp.2, (2016) DOI: 10.1155/2016/7954154

[10] J. Arevalo, F. A. Gonzalez, R. Ramos-Pollan, J. L. Oliveira, and M. A. G. Lopez, "Convolutional neural networks for mammography mass lesion classification," in Proceedings of the 37th Annual International Conference of the IEEE Engineering in Medicine and Biology Society (EMBC '15), pp.797-800, August (2015) https://ieeexplore.ieee.org/document/7318482 
[11] Jia Y., Shelhamer E., Donahue J., Karayev S., Long J., Girshick R., Guadarrama S., and Darrell T., Caffe: Convolutional architecture for fast feature embedding, in: Proceedings of the 22nd ACM international conference on Multimedia, ACM. pp.675-678, (2014) DOI: 10.1145/2647868.2654889

[12] Kawasaki Y., Uga H., Kagiwada S., Iyatomi H., "Basic study of automated diagnosis of viral plant diseases using convolutional neural networks," in: International Symposium on Visual Computing, Springer, pp.638645, (2015) DOI: 10.14864/fss.31.0_391

[13] Brahimi M., Boukhalfa K., Moussaoui A., "Deep learning for tomato diseases: classification and symptoms visualization," Applied Artificial Intelligence, vol.31, pp.299-315, (2017) DOI: 10.1080/08839514.201 7.1315516

[14] DeChant C., Wiesner Hanks T., Chen S., Stewart E. L., Yosinski J., Gore M. A., Nelson R. J., Lipson H., Automated identification of northern leaf blight-infected maize plants from field imagery using deep learning. Phytopathology, vol.107,1426-1432, (2017) DOI: 10.1094/PHYTO-11-16-0417-R

[15] Brahimi M., Arsenovic M., Laraba S., Sladojevic S., Boukhalfa K., and Moussaoui A., "Deep learning for plant diseases: Detection and saliency map visualisation, in: Human and Machine Learning," Springer, pp.93-117, (2018) DOI: 10.1007/978-3-319-90403-0_6

[16] Wang J., Chen L., Zhang J., Yuan Y., Li M., and ZengW., "Cnn transfer learning for automatic image-based classification of crop disease, in: Chinese Conference on Image and Graphics Technologies," Springer. pp.319-329, https://link.springer.com/chapter/10.1007\%2F978-981-13-1702-6_32, (2018)

[17] Pic'o, B., D'1ez, M.J., Nuez, F., "Viral diseases causing the greatest economic losses to the tomato crop," II. the tomato yellow leaf curl virus-a review, Scientia Horticulturae, vol.67, pp.151-196, (1996) DOI: 10.1016/S0304-4238(96)00945-4

[18] Agarwal M., Singh A., Arjaria S., Sinha A., and Gupta S. "ToLeD: Tomato leaf disease detection using convolution neural network," Procedia Computer Science, vol.167, pp.293-301. DOI: 10.1016/j.procs.202 0.03 .225

\section{Authors}

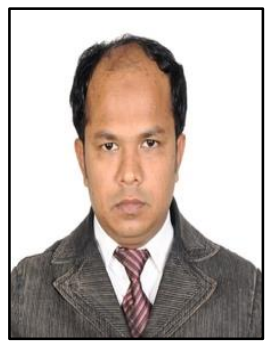

\section{Md Abdur Rahman}

Md. A. Rahman was born in Bangladesh, in 1978. Since 2002, he has been currently working as an Associate Professor of Computer Science at the department of Mathematics, Jahangirnagar, University, Bangladesh. He received B.Sc. degree from same university in 2001, and MS in Computational Science from Texas A\&M University-Commerce, TX, USA, in 2014. From 2012 to 2014, he was a Graduate Teaching Assistant at Computer Science and Information Systems in Texas A\&M UniversityCommerce, TX, USA. His specialization is on Intelligent Information Systems (IIS), and research interests include multi-agent path planning (robotics), deep learning, smart agriculture, and networking. Also, he worked to develop single page web application for several prestigious projects of United States of America (USA) using ANGULARJS, J2EE, SPRING, NODEJS, MYSQL, etc.; these sound experience helps him to handle different type of projects in an excellent way. Currently, Mr. Rahman is focusing to do research on multiagent path planning, and smart agriculture for classification of plant diseases using DEEP LEARNING (python, Keras). Moreover, Mr. Rahman is working as an executive member of International Conference on Information Technology (www.icit.org) since 2018. 